PROCEEDINGS OF THE

AMERICAN MATHEMATICAL SOCIETY

Volume 132, Number 5, Pages 1435-1443

S 0002-9939(03)07220-4

Article electronically published on September 18, 2003

\title{
A FIXED POINT THEOREM IN PARTIALLY ORDERED SETS AND SOME APPLICATIONS TO MATRIX EQUATIONS
}

\author{
ANDRÉ C. M. RAN AND MARTINE C. B. REURINGS
}

(Communicated by Joseph A. Ball)

\begin{abstract}
An analogue of Banach's fixed point theorem in partially ordered sets is proved in this paper, and several applications to linear and nonlinear matrix equations are discussed.
\end{abstract}

\section{INTRODUCTION}

In this paper we discuss an analogue of Banach's fixed point theorem in partially ordered sets and several applications. The key feature in this fixed point theorem is that the contractivity condition on the nonlinear map is only assumed to hold on elements that are comparable in the partial order. However, the map is assumed to be monotone. We show that under such conditions the conclusions of Banach's fixed point theorem still hold.

It should be noted that there are many fixed point theorems for order-preserving or order-reversing maps on lattices. See [12, [1, 2. However, for order-preserving maps the assumption is usually that the lattice is complete, which implies, for instance, that there is a maximal element in the lattice. Since the applications we have in mind concern the lattice of Hermitian matrices or the cone of positive definite matrices, this does not hold for some of our applications. For order-reversing maps there are usually conditions that imply that there cannot be a periodic orbit of period two. For our applications such assumptions are either not true or hard to check.

In Sections 3 and 4 we discuss several applications. Section 3 concerns linear matrix equations of the type

$$
X-A_{1}^{*} X A_{1}-\cdots-A_{m}^{*} X A_{m}=Q
$$

and

$$
X+A_{1}^{*} X A_{1}+\cdots+A_{m}^{*} X A_{m}=Q,
$$

where $Q$ is a positive definite matrix and $A_{1}, \ldots, A_{m}$ are arbitrary $n \times n$ matrices. Our main result in this section gives a condition under which these equations have a unique Hermitian solution. In this way we derive by totally different means some of the main results of [7. Compare also [11].

Received by the editors June 19, 2002 and, in revised form, January 8, 2003.

2000 Mathematics Subject Classification. Primary 47H10; Secondary 15A24, 54H25.

(C)2003 American Mathematical Society 
Section 4 concerns general nonlinear matrix equations of the type

$$
X=Q \pm \sum_{j=1}^{m} A_{j}^{*} \mathcal{F}(X) A_{j},
$$

where again $Q$ is a positive definite matrix and $A_{1}, \ldots, A_{m}$ are arbitrary $n \times n$ matrices, and where $\mathcal{F}$ is a continuous and monotone map from the set of positive definite matrices to itself. As a particular case we study an equation originating from Chapter 7 in [10, which closely resembles the discrete algebraic Riccati equation.

\section{A FIXED POINT THEOREM ON PARTIALLY ORDERED SETS}

In this section we will prove the following fixed point theorem, which, to the best of our knowledge, is new.

Theorem 2.1. Let $T$ be a partially ordered set such that every pair $x, y \in T$ has a lower bound and an upper bound. Furthermore, let $d$ be a metric on $T$ such that $(T, d)$ is a complete metric space. If $\mathcal{F}$ is a continuous, monotone (i.e., either order-preserving or order-reversing) map from $T$ into $T$ such that

$$
\begin{gathered}
\exists 0<c<1: d(\mathcal{F}(x), \mathcal{F}(y)) \leq c d(x, y), \quad \forall x \geq y, \\
\exists x_{0} \in T: x_{0} \leq \mathcal{F}\left(x_{0}\right) \text { or } x_{0} \geq \mathcal{F}\left(x_{0}\right),
\end{gathered}
$$

then $\mathcal{F}$ has a unique fixed point $\bar{x}$. Moreover, for every $x \in T$,

$$
\lim _{n \rightarrow \infty} \mathcal{F}^{n}(x)=\bar{x} \text {. }
$$

Proof. Let $x_{0} \in T$ be such that $x_{0} \leq \mathcal{F}\left(x_{0}\right)$ or $x_{0} \geq \mathcal{F}\left(x_{0}\right)$. The monotonicity of $\mathcal{F}$ implies that either $\mathcal{F}^{n}\left(x_{0}\right) \leq \mathcal{F}^{n+1}\left(x_{0}\right)$ or $\mathcal{F}^{n}\left(x_{0}\right) \geq \mathcal{F}^{n+1}\left(x_{0}\right)$ for $n=0,1,2, \ldots$ So from (11) it follows that

$$
d\left(\mathcal{F}^{n+1}\left(x_{0}\right), \mathcal{F}^{n}\left(x_{0}\right)\right) \leq c d\left(\mathcal{F}^{n}\left(x_{0}\right), \mathcal{F}^{n-1}\left(x_{0}\right)\right) .
$$

Hence, induction gives

$$
d\left(\mathcal{F}^{n+1}\left(x_{0}\right), \mathcal{F}^{n}\left(x_{0}\right)\right) \leq c^{n} d\left(\mathcal{F}\left(x_{0}\right), x_{0}\right) .
$$

At this point we follow the proof of Banach's fixed point theorem. So now we will prove that $\left\{\mathcal{F}^{n}\left(x_{0}\right)\right\}_{n=0}^{\infty}$ is a Cauchy sequence. Let $n<m$. Then

$$
\begin{aligned}
d\left(\mathcal{F}^{n}\left(x_{0}\right), \mathcal{F}^{m}\left(x_{0}\right)\right) & \leq \sum_{i=n+1}^{m} d\left(\mathcal{F}^{i}\left(x_{0}\right), \mathcal{F}^{i-1}\left(x_{0}\right)\right) \\
& \leq\left(c^{n}+c^{n+1}+\cdots+c^{m-n-1}\right) d\left(\mathcal{F}\left(x_{0}\right), x_{0}\right) \\
& =c^{n} \frac{1-c^{m-n-1}}{1-c} d\left(\mathcal{F}\left(x_{0}\right), x_{0}\right) .
\end{aligned}
$$

So $\left\{\mathcal{F}^{n}\left(x_{0}\right)\right\}_{n=0}^{\infty}$ is indeed a Cauchy sequence. Since $T$ is complete, it follows that

$$
\lim _{n \rightarrow \infty} \mathcal{F}^{n}\left(x_{0}\right)=\bar{x}
$$

for some $\bar{x} \in T$. Because $\mathcal{F}$ is continuous, $\bar{x}$ is a fixed point of $\mathcal{F}$.

It remains for us to show that $\bar{x}$ is the unique fixed point of $\mathcal{F}$. We will do this by showing that $\lim _{n \rightarrow \infty} \mathcal{F}^{n}(x)=\bar{x}$ for every $x \in T$. Thus, for $x \leq x_{0}$ and $x \geq x_{0}$ it is obvious, because in both cases $\mathcal{F}^{n}(x) \leq \mathcal{F}^{n}\left(x_{0}\right)$ or $\mathcal{F}^{n}(x) \geq \mathcal{F}^{n}\left(x_{0}\right)$. Hence, 
with (11) we see that $d\left(\mathcal{F}^{n}(x), \mathcal{F}^{n}\left(x_{0}\right)\right) \leq c^{n} d\left(x, x_{0}\right)$. Note that the right-hand side tends to 0 if $n \rightarrow \infty$. So

$$
\lim _{n \rightarrow \infty} \mathcal{F}^{n}(x)=\lim _{n \rightarrow \infty} \mathcal{F}^{n}\left(x_{0}\right)=\bar{x} .
$$

Finally, let $x \in T$ be arbitrary and let $x_{1}$, resp. $x_{2}$, be an upper bound, resp. a lower bound, of $x$ and $x_{0}$. Then

$$
x_{1} \geq x \geq x_{2} \quad \text { and } \quad x_{1} \geq x_{0} \geq x_{2} .
$$

From (3) it follows that

$$
\mathcal{F}^{n}\left(x_{1}\right) \geq \mathcal{F}^{n}(x) \geq \mathcal{F}^{n}\left(x_{2}\right) \quad \text { or } \quad \mathcal{F}^{n}\left(x_{1}\right) \leq \mathcal{F}^{n}(x) \leq \mathcal{F}^{n}\left(x_{2}\right)
$$

and

$$
\lim _{n \rightarrow \infty} \mathcal{F}^{n}\left(x_{1}\right)=\lim _{n \rightarrow \infty} \mathcal{F}^{n}\left(x_{2}\right)=\bar{x} .
$$

By combining (4) and (5) we get $\lim _{n \rightarrow \infty} \mathcal{F}^{n}(x)=\bar{x}$. This proves the theorem.

Recall that Banach's fixed point theorem states that $\mathcal{F}$ has a unique fixed point if $\mathcal{F}$ maps a complete metric space $S$ into itself and satisfies

$$
\exists 0<c<1: d(\mathcal{F}(x), \mathcal{F}(y)) \leq c d(x, y), \quad \forall x, y \in S .
$$

Observe that this condition is slightly stronger than condition (1) of Theorem 2.1

\section{Application to linear matrix equations}

We shall apply this fixed point theorem to linear matrix equations of the type

$$
X-A_{1}^{*} X A_{1}-\cdots-A_{m}^{*} X A_{m}=Q
$$

and

$$
X+A_{1}^{*} X A_{1}+\cdots+A_{m}^{*} X A_{m}=Q
$$

where $Q$ is a positive definite matrix and $A_{1}, \ldots, A_{m}$ are arbitrary $n \times n$ matrices. We are particularly interested in (unique) positive definite solutions. These equations were studied in detail in 7]. The results we shall derive below were obtained there by two methods which are totally different from the method we will use in this paper. The first method in [7] is based on the fact that (6) is equivalent to the matrix equation $G x=q$, where $G=\sum_{j=1}^{m} A_{i}^{T} \otimes A_{i}^{*}$ and where $q$ is a vector constructed from the elements of $Q$; see Chapter 12 in [6]. The second method involves results from the theory of operators mapping a positive cone into itself. To be precise, the results can be deduced from the main result in [11.

We shall use the following notation: $\mathcal{M}(n)$ denotes the set of all $n \times n$ matrices, $\mathcal{H}(n) \subset \mathcal{M}(n)$ the set of all $n \times n$ Hermitian matrices and $\mathcal{P}(n) \subset \mathcal{H}(n)$ is the set of all $n \times n$ positive definite matrices. Instead of $X \in \mathcal{P}(n)$ we will also write $X>0$. Furthermore, $X \geq 0$ means that $X$ is positive semidefinite. As a different notation 
for $X-Y \geq 0(X-Y>0)$ we will use $X \geq Y(X>Y)$. If $X, Y \in \mathcal{H}(n)$ such that $X \leq Y$, then $[X, Y]$ will be the set of all $Z \in \mathcal{H}(n)$ satisfying $X \leq Z \leq Y$. We denote by $\|\cdot\|$ the spectral norm, i.e., $\|A\|=\sqrt{\lambda^{+}\left(A^{*} A\right)}$ where $\lambda^{+}\left(A^{*} A\right)$ is the largest eigenvalue of $A^{*} A$. The $n \times n$ identity matrix will be written as $I_{n}$.

Define maps $\mathcal{G}$ and $\mathcal{K}$ on $\mathcal{H}(n)$ by

$$
\mathcal{G}(X)=Q+\sum_{j=1}^{m} A_{j}^{*} X A_{j}, \quad \mathcal{K}(X)=Q-\sum_{j=1}^{m} A_{j}^{*} X A_{j} .
$$

Note that the fixed points of $\mathcal{G}$ are the solutions of $[\underline{6}$, while the fixed points of $\mathcal{K}$ are the solutions of (7).

Most of the conditions of Theorem 2.1 are satisfied for $\mathcal{G}$ and $\mathcal{K}$. Indeed, $\mathcal{G}$ and $\mathcal{K}$ both are monotone and continuous. The set $\mathcal{H}(n)$ is partially ordered and for every $X, Y \in \mathcal{H}(n)$ there is a greatest lower bound and a least upper bound. Condition (2) is satisfied for $x_{0}=0$, since $\mathcal{G}(0)=\mathcal{K}(0)=Q>0$. So we only have to derive a condition on $\mathcal{G}$ and $\mathcal{K}$ such that (1) is fulfilled. For this we also have to specify which metric we shall use on $\mathcal{H}(n)$. It turns out that it is convenient here to use the metric induced by the trace norm $\|\cdot\|_{1}$. Recall that this norm is given by $\|A\|_{1}=\sum_{j=1}^{n} s_{j}(A)$, where $s_{j}(A), j=1, \ldots, n$ are the singular values of $A$. In fact, we shall use a slight modification of this norm. For $Q \in \mathcal{P}(n)$ we define $\|A\|_{1, Q}=\left\|Q^{\frac{1}{2}} A Q^{\frac{1}{2}}\right\|_{1}$. This again defines a norm, and from Theorem IX.2.2 in [4] we know that $\mathcal{H}(n)$ equipped with the metric induced by $\|\cdot\|_{1, Q}$ is a complete metric space for any positive definite $Q$.

The following lemma is useful for our application.

Lemma 3.1. Let $A \geq 0$ and $B \geq 0$ be $n \times n$ matrices. Then $0 \leq \operatorname{tr}(A B) \leq$ $\|A\| \cdot \operatorname{tr}(B)$.

Proof. It is well known (see Exercise 3 on p. 468 in [5]) that the eigenvalues of the product of two positive semidefinite matrices are nonnegative. In particular, $\operatorname{tr}(A B) \geq 0$. Furthermore, since $A \leq\|A\| I_{n}$, we have

$$
0 \leq \operatorname{tr}((\|A\|-A) B)=\operatorname{tr}(\|A\| B-A B)=\|A\| \operatorname{tr}(B)-\operatorname{tr}(A B)
$$

which completes the proof.

Theorem 3.1. Let $Q \in \mathcal{P}(n)$ and let $\mathcal{K}(\tilde{Q})>0$ for some $\tilde{Q} \in \mathcal{P}(n)$. Then

(1) $\mathcal{G}$ and $\mathcal{K}$ have a unique fixed point in $\mathcal{H}(n)$.

(2) For any $X_{0} \in \mathcal{H}(n)$, the sequence $\left\{\mathcal{G}^{j}\left(X_{0}\right)\right\}_{j=0}^{\infty}$ converges to the solution of (6) which is positive definite, while the sequence $\left\{\mathcal{K}^{j}\left(X_{0}\right)\right\}_{j=0}^{\infty}$ converges to the solution of (7).

(3) If, in addition, $\mathcal{K}(Q)>0$, then the unique solution of (7) is in the set $[\mathcal{K}(Q), Q]$. In particular, it is positive definite. 
Proof. We will only consider $\mathcal{G}$. The proof for $\mathcal{K}$ is the same. Let $X, Y \in \mathcal{H}(n)$ such that $X \leq Y$. Then $\mathcal{G}(X) \leq \mathcal{G}(Y)$. Therefore,

$$
\begin{aligned}
& \|\mathcal{G}(Y)-\mathcal{G}(X)\|_{1, \tilde{Q}}=\operatorname{tr}\left(\tilde{Q}^{\frac{1}{2}}(\mathcal{G}(Y)-\mathcal{G}(X)) \tilde{Q}^{\frac{1}{2}}\right) \\
& =\operatorname{tr}\left(\sum_{j=1}^{m} \tilde{Q}^{\frac{1}{2}} A_{j}^{*}(Y-X) A_{j} \tilde{Q}^{\frac{1}{2}}\right)=\sum_{j=1}^{m} \operatorname{tr}\left(\tilde{Q}^{\frac{1}{2}} A_{j}^{*}(Y-X) A_{j} \tilde{Q}^{\frac{1}{2}}\right) \\
& =\sum_{j=1}^{m} \operatorname{tr}\left(A_{j} \tilde{Q} A_{j}^{*}(Y-X)\right)=\sum_{j=1}^{m} \operatorname{tr}\left(A_{j} \tilde{Q} A_{j}^{*} \tilde{Q}^{-\frac{1}{2}} \tilde{Q}^{\frac{1}{2}}(Y-X) \tilde{Q}^{\frac{1}{2}} \tilde{Q}^{-\frac{1}{2}}\right) \\
& =\sum_{j=1}^{m} \operatorname{tr}\left(\tilde{Q}^{-\frac{1}{2}} A_{j} \tilde{Q} A_{j}^{*} \tilde{Q}^{-\frac{1}{2}} \tilde{Q}^{\frac{1}{2}}(Y-X) \tilde{Q}^{\frac{1}{2}}\right) \\
& =\operatorname{tr}\left(\sum_{j=1}^{m} \tilde{Q}^{-\frac{1}{2}} A_{j} \tilde{Q} A_{j}^{*} \tilde{Q}^{-\frac{1}{2}} \tilde{Q}^{\frac{1}{2}}(Y-X) \tilde{Q}^{\frac{1}{2}}\right) \\
& =\operatorname{tr}\left(\left(\sum_{j=1}^{m} \tilde{Q}^{-\frac{1}{2}} A_{j} \tilde{Q} A_{j}^{*} \tilde{Q}^{-\frac{1}{2}}\right)\left(\tilde{Q}^{\frac{1}{2}}(Y-X) \tilde{Q}^{\frac{1}{2}}\right)\right) \\
& \leq\left\|\sum_{j=1}^{m} \tilde{Q}^{-\frac{1}{2}} A_{j} \tilde{Q} A_{j}^{*} \tilde{Q}^{-\frac{1}{2}}\right\| \cdot\|Y-X\|_{1, \tilde{Q}} \cdot
\end{aligned}
$$

The inequality follows from Lemma [3.1. Because of the assumption that $0 \leq$ $\sum_{j=1}^{m} A_{j} \tilde{Q} A_{j}^{*}<\tilde{Q}$, condition (11) of Theorem 2.1] is satisfied with

$$
c=\left\|\sum_{j=1}^{m} \tilde{Q}^{-\frac{1}{2}} A_{j} \tilde{Q} A_{j}^{*} \tilde{Q}^{-\frac{1}{2}}\right\| .
$$

So we can apply that theorem to get the first part of the theorem.

To show that the solution of (6) is positive definite, just observe that $\mathcal{G}$ maps $\mathcal{P}(n)$ into the set $\{X \in \mathcal{H}(n) \mid X \geq Q\}$. So the solution must be in this set, and hence it is positive definite.

Next we consider the solution of (77) under the additional assumption that $\mathcal{K}(Q)>$ 0 . Note that $\mathcal{K}$ is order-reversing. Also, we have that $0<\mathcal{K}(Q) \leq Q$. It is then easily seen that $\mathcal{K}$ maps the compact convex set $[\mathcal{K}(Q), Q]$ into itself. Since $\mathcal{K}$ is continuous, it follows from Schauder's fixed point theorem that $\mathcal{K}$ has at least one fixed point in this set. However, fixed points of $\mathcal{K}$ are solutions of (7), and we proved already that (7) has a unique Hermitian solution. Thus this solution must be in the set $[\mathcal{K}(Q), Q]$.

\section{Applications to nOnlinear matrix equations}

In this section we study the following class of nonlinear matrix equations:

$$
X=Q \pm \sum_{j=1}^{m} A_{j}^{*} \mathcal{F}(X) A_{j} .
$$

Here, again $Q$ is positive definite and the $A_{j}$ are abitrary $n \times n$ matrices, and $\mathcal{F}$ is a continuous map, mapping $\mathcal{P}(n)$ into $\mathcal{P}(n)$. We shall also assume that $\mathcal{F}$ is 
either order-preserving or order-reversing. The map $X \rightarrow Q \pm \sum_{j=1}^{m} A_{j}^{*} \mathcal{F}(X) A_{j}$ is denoted by $\mathcal{G}$.

In total this gives us four cases to consider, some of which will require extra assumptions, mainly because the map $\mathcal{G}$ may end up giving nonpositive values for some positive definite matrices $X$.

For the case $m=1$, see [3].

The present case is motivated by Chapter 7 of [10], where the following matrix equation appears:

$$
X=Q-A^{*}(D-\tilde{X})^{-1} A .
$$

Here $A$ is the $m n \times n$ block matrix given by

$$
A=\left(\begin{array}{c}
A_{1} \\
\vdots \\
A_{m}
\end{array}\right)
$$

where each of the matrices $A_{j}$ is an $n \times n$ matrix. Moreover, $Q>0$ is an $n \times n$ matrix, and $D \geq 0$ is an $n m \times n m$ matrix, while $\tilde{X}$ is the $m \times m$ block-diagonal matrix with the $n \times n$ matrix $X$ on each diagonal entry. In case $D$ is block diagonal as well with the same matrix on each block diagonal entry, i.e., $D=\tilde{C}$ for some $C$, the equation can be rewritten as

$$
X=Q+\sum_{j=1}^{m} A_{j}^{*}(X-C)^{-1} A_{j},
$$

which is of the form (8) with an order-reversing map $\mathcal{F}$ as long as $X>C$.

4.1. Case 1. In this subsection we consider the equation

$$
X=Q+\sum_{j=1}^{m} A_{j}^{*} \mathcal{F}(X) A_{j},
$$

with $\mathcal{F}$ being order-preserving. In that case $\mathcal{G}$ is well defined on $\mathcal{P}(n)$ and orderpreserving as well. Since $\mathcal{G}(X) \geq Q$ for all $X \in \mathcal{P}(n)$, it is easily seen that the sequence $\left\{\mathcal{G}^{j}(Q)\right\}_{j=0}^{\infty}$ is an increasing sequence. In particular, $\mathcal{G}(Q) \geq Q$. So condition (2) in Theorem 2.1 is satisfied.

Our first result discusses existence of a solution.

Proposition 4.1. Assume that there exists an $X_{0}$ such that $\mathcal{G}\left(X_{0}\right) \leq X_{0}$. Then $\mathcal{G}$ maps the set $\left[Q, X_{0}\right]$ into itself, the limit $X_{-}=\lim _{j \rightarrow \infty} \mathcal{G}^{j}(Q)$ exists and is the smallest solution to (11). Moreover, the sequence $\left\{\mathcal{G}^{j}\left(X_{0}\right)\right\}_{j=0}^{\infty}$ decreases to a solution $X_{+}$, which is the largest solution in the set $\left[Q, X_{0}\right]$.

Proof. Since $\mathcal{G}\left(X_{0}\right) \leq X_{0}$, we have $Q \leq \mathcal{G}\left(X_{0}\right) \leq X_{0}$. Now if $Q \leq X \leq X_{0}$, we easily see that $Q \leq \mathcal{G}(Q) \leq \mathcal{G}(X) \leq \mathcal{G}\left(X_{0}\right) \leq X_{0}$.

Applying $\mathcal{G}$ repeatedly, we see that the sequence $\left\{\mathcal{G}^{j}(Q)\right\}_{j=0}^{\infty}$ is an increasing sequence, bounded above by $\mathcal{G}^{l}\left(X_{0}\right)$ for any value of $l$. Also, the sequence $\left\{\mathcal{G}^{j}\left(X_{0}\right)\right\}_{j=0}^{\infty}$ is a decreasing sequence bounded below. Thus, both sequences converge.

Now let $X$ be any solution to the equation (11). Then $Q \leq X=\mathcal{G}(X)$, and by repeatedly applying $\mathcal{G}$ we see that $X_{-} \leq X$. If $X \in\left[Q, X_{0}\right]$, i.e., if $X \leq X_{0}$, we see, again by applying $\mathcal{G}$ repeatedly, that $X \leq X_{+}$. 
To get uniqueness of the solution, we can follow the same line of argument as in the proof of Theorem 3.1 to derive the following result. For the sake of completeness we shall provide the proof in this case.

Theorem 4.1. Let $Q \in \mathcal{P}(n)$. Assume there is a positive number $M$ for which $\sum_{j=1}^{m} A_{j} A_{j}^{*}<M \cdot I_{n}$ and such that for $X \leq Y$ we have

$$
|\operatorname{tr}(\mathcal{F}(Y)-\mathcal{F}(X))| \leq \frac{1}{M}|\operatorname{tr}(Y-X)|
$$

Then equation (11) has a unique solution in $\mathcal{P}(n)$.

Proof. Let $X, Y \in \mathcal{H}(n)$ such that $X \leq Y$. Then $\mathcal{G}(X) \leq \mathcal{G}(Y)$. Therefore,

$$
\begin{aligned}
\| \mathcal{G}(Y) & -\mathcal{G}(X) \|_{1}=\operatorname{tr}(\mathcal{G}(Y)-\mathcal{G}(X))=\operatorname{tr}\left(\sum_{j=1}^{m} A_{j}^{*}(\mathcal{F}(Y)-\mathcal{F}(X)) A_{j}\right) \\
& =\sum_{j=1}^{m} \operatorname{tr}\left(A_{j}^{*}(\mathcal{F}(Y)-\mathcal{F}(X)) A_{j}\right)=\sum_{j=1}^{m} \operatorname{tr}\left(A_{j} A_{j}^{*}(\mathcal{F}(Y)-\mathcal{F}(X))\right) \\
& =\operatorname{tr}\left(\sum_{j=1}^{m} A_{j} A_{j}^{*}(\mathcal{F}(Y)-\mathcal{F}(X))\right)=\operatorname{tr}\left(\left(\sum_{j=1}^{m} A_{j} A_{j}^{*}\right)(\mathcal{F}(Y)-\mathcal{F}(X))\right) \\
& \leq\left\|\sum_{j=1}^{m} A_{j} A_{j}^{*}\right\|\|\mathcal{F}(Y)-\mathcal{F}(X)\|_{1} .
\end{aligned}
$$

As before, the inequality follows from Lemma 3.1. Because of the assumptions in the theorem we see that condition (1) of Theorem 2.1 is satisfied. So we can apply that theorem to get the desired result.

4.2. Case 2. Next, we consider (11) under the assumption that $\mathcal{F}$ is decreasing. Then also $\mathcal{G}$ is decreasing, but still well defined on $\mathcal{P}(n)$. One easily sees that $\mathcal{G}^{2}(X) \in[Q, \mathcal{G}(Q)]$ for every positive definite $X$. In particular, this set is mapped into itself. As in [3] one checks that the sequence $\left\{\mathcal{G}^{2 j}(Q)\right\}_{j=0}^{\infty}$ increases to a limit $X_{-\infty}$, while the sequence $\left\{\mathcal{G}^{2 j+1}\right\}_{j=0}^{\infty}$ decreases to a limit $X_{+\infty}$. These two matrices form a periodic orbit of period two, and any periodic orbit (including fixed points of $\mathcal{G}$, of which there is at least one), is contained in $\left[X_{-\infty}, X_{+\infty}\right]$. In this case Theorem 4.1 holds as well, but the condition can be weakened a bit, since we know in advance that a fixed point exists and must be contained in the set $[Q, \mathcal{G}(Q)]$.

Theorem 4.2. Let $Q \in \mathcal{P}(n)$. Assume there is a positive number $M$ for which $\sum_{j=1}^{m} A_{j} A_{j}^{*}<M \cdot I_{n}$ and such that for $Q \leq X \leq Y \leq \mathcal{G}(Q)$ we have

$$
|\operatorname{tr}(\mathcal{F}(Y)-\mathcal{F}(X))| \leq \frac{1}{M}|\operatorname{tr}(Y-X)| \text {. }
$$

Then equation (11) has a unique solution in $\mathcal{P}(n)$.

We return to equation (10), which falls in this case. We shall assume that $C<Q$. The map $\mathcal{G}$ is given by $\mathcal{G}(X)=Q+\sum_{j=1}^{m} A_{j}^{*}(X-C)^{-1} A_{j}$.

Then we have the following theorem (compare [10], Theorem 7.1.2, which gives a similar, but unfortunately wrong result). 
Theorem 4.3. Suppose that $\left\|\sum_{j=1}^{m} A_{j} A_{j}^{*}\right\| \cdot\left\|(Q-C)^{-1}\right\|^{2}<1$. Then (10) has a unique solution, and for any $X_{0}>Q$ the sequence $\left\{\mathcal{G}^{j}\left(X_{0}\right)\right\}_{j=0}^{\infty}$ converges to the unique solution.

Proof. Observe that

$$
(X-C)^{-1}-(Y-C)^{-1}=(X-C)^{-1}(Y-X)(Y-C)^{-1} .
$$

Hence

$$
\left|\operatorname{tr}\left((X-C)^{-1}-(Y-C)^{-1}\right)\right| \leq\left\|(X-C)^{-1}\right\| \cdot\left\|(Y-C)^{-1}\right\| \cdot|\operatorname{tr}(Y-X)| .
$$

In case $X>Q$ and $Y>Q$ we have, since $C<Q$, that $\left\|(X-C)^{-1}\right\| \leq\left\|(Q-C)^{-1}\right\|$ and $\left\|(Y-C)^{-1}\right\| \leq\left\|(Q-C)^{-1}\right\|$. The theorem is now a straightforward consequence of the previous one and of Theorem 2.1 .

It turns out that equation (9) has a unique solution under much less stringent conditions, and that the iteration of $\mathcal{G}$ on starting values $X_{0}>Q$ will converge under these less stringent conditions. This will be proven in a forthcoming publication [8]. The techniques for proving this are quite different from the ones used here.

4.3. Case 3. In this and in the next subsection we consider the equation

$$
X=Q-\sum_{j=1}^{m} A_{j}^{*} \mathcal{F}(X) A_{j} .
$$

In this subsection $\mathcal{F}$ will be taken to be order-preserving. Now we have to be careful with iterations of $\mathcal{G}$ since $\mathcal{G}(X)$ may turn out not to be positive definite any more. We shall assume that $\mathcal{G}(Q)>0$. Clearly, $\mathcal{G}(X) \leq Q$ for all $X \in \mathcal{P}(n)$. Since $\mathcal{G}$ is now order-reversing, we see that for $0 \leq X \leq Q$ we have that $\mathcal{G}(Q) \leq \mathcal{G}(X) \leq Q$. In particular, again the set $[\mathcal{G}(Q), Q]$ is mapped into itself, and any fixed point of $\mathcal{G}$ must be in this set. As in the case treated in the previous subsection we obtain a periodic orbit of period two from limits of $\mathcal{G}^{2 j}(Q)$ and $\mathcal{G}^{2 j+1}(Q)$. As an analogue of Theorem 4.1 we have the following result. Note that condition (2) in Theorem 2.1 is again satisfied for $Q$.

Theorem 4.4. Let $Q \in \mathcal{P}(n)$. Assume that $\mathcal{G}(Q)>0$. Furthermore, assume there is a positive number $M$ for which $\sum_{j=1}^{m} A_{j} A_{j}^{*}<M \cdot I_{n}$ and such that for $\mathcal{G}(Q) \leq X \leq Y \leq Q$ we have

$$
|\operatorname{tr}(\mathcal{F}(Y)-\mathcal{F}(X))| \leq \frac{1}{M}|\operatorname{tr}(Y-X)| .
$$

Then equation (12) has a unique solution in $\mathcal{P}(n)$.

4.4. Case 4. Finally, in this subsection we consider again equation (12), but with $\mathcal{F}$ order-reversing. Then $\mathcal{G}$ is order-preserving. Clearly, again all values of $\mathcal{G}$ are matrices in $(0, Q]$. Again, we have to be careful with the iteration of $\mathcal{G}$. For this purpose we shall assume that there is an $X_{0} \leq Q$ such that $X_{0} \leq \mathcal{G}\left(X_{0}\right)$. Then we have

$$
X_{0} \leq \mathcal{G}\left(X_{0}\right) \leq \mathcal{G}(Q) \leq Q
$$

One easily sees from this that $\left[X_{0}, Q\right]$ is mapped into itself, that $\left\{\mathcal{G}^{j}\left(X_{0}\right)\right\}_{j=0}^{\infty}$ is an increasing sequence, and that $\left\{\mathcal{G}^{j}(Q)\right\}_{j=0}^{\infty}$ is a decreasing sequence. Moreover, for any $j$ and $l$ we have $\mathcal{G}^{j}\left(X_{0}\right) \leq \mathcal{G}^{l}(Q)$. Hence both sequences have a limit, which we shall denote by $X_{-}$and $X_{+}$, respectively. We have the following proposition, which 
is the analogue of Proposition 4.1. The proof is virtually the same, and hence will be omitted.

Proposition 4.2. Assume that there exists an $X_{0}$ such that $\mathcal{G}\left(X_{0}\right) \geq X_{0}$. Then $\mathcal{G}$ maps the set $\left[X_{0}, Q\right]$ into itself, the limit $X_{+}=\lim _{j \rightarrow \infty} \mathcal{G}^{j}(Q)$ exists and is the largest solution to (12). Moreover, the sequence $\left\{\mathcal{G}^{j}\left(X_{0}\right)\right\}_{j=0}^{\infty}$ increases to a solution $X_{-}$, which is the smallest solution in the set $\left[X_{0}, Q\right]$.

As an analogue of Theorem 4.1 we have the following result. Again, the proof is a straightforward application of Theorem 2.1 and hence will be omitted.

Theorem 4.5. Let $Q \in \mathcal{P}(n)$. Assume that there exists an $X_{0} \leq Q$ such that $\mathcal{G}\left(X_{0}\right) \geq X_{0}$. Furthermore, assume that there is a positive number $M$ for which $\sum_{j=1}^{m} A_{j} A_{j}^{*}<M \cdot I_{n}$ and such that for $X_{0} \leq X \leq Y \leq Q$ we have

$$
|\operatorname{tr}(\mathcal{F}(Y)-\mathcal{F}(X))| \leq \frac{1}{M}|\operatorname{tr}(Y-X)| .
$$

Then equation (12) has a unique solution in $\left[X_{0}, Q\right]$.

\section{REFERENCES}

[1] A. Björner, Order-reversing maps and unique fixed points in complete lattices, Algebra Universalis, 12:402-403, 1981. MR 82i:06006

[2] C. Blair and A. E. Roth, An extension and simple proof of a constrained lattice fixed point theorem, Algebra Universalis, 9:131-132, 1979. Erratum, ibid, 12:134, 1981. MR 80b:06009. MR 82c:06016

[3] S. M. El-Sayed and A. C. M. Ran, On an iteration method for solving a class of nonlinear matrix equations, SIAM J. Matrix Anal. Appl., 23:632-645, 2002. MR 2002m:15023

[4] I. Gohberg and S. Goldberg, Basic Operator Theory, Birkhäuser, Boston, MA, 1981. MR 83b:47001

[5] R. A. Horn and C. R. Johnson, Matrix Analysis, Cambridge University Press, Cambridge, 1985. MR 87e:15001

[6] P. Lancaster and M. Tismenetsky, The Theory of Matrices, Second edition, Academic Press, Inc., Orlando, FL, 1985. MR 87a:15001

[7] M. C. B. Reurings and A. C. M. Ran. The Symmetric Linear Matrix Equation, Electronic Journal of Linear Algebra 9 (2002), 93-107.

[8] M. C. B. Reurings and A. C. M. Ran, A nonlinear matrix equation connected to interpolation theory. Linear Algebra and its Applications, to appear.

[9] P. Rózsa, Lineare Matrizengleichungen und Kroneckersche Produkte, Zeitschrift fur angewandte Mathematik und Mechanik, 58:T395-T397, 1978. MR 58:22115

[10] L. A. Sakhnovich, Interpolation theory and its applications, Kluwer, Dordrecht, 1997. MR 99j:47016

[11] H. Schneider, Positive Operators and an Inertia Theorem, Numerische Mathematik 7:11-17, 1965. MR 30:3888

[12] A. Tarski, A lattice-theoretical fixpoint theorem and its applications, Pacific Journal of Math. 5:285-309, 1955. MR 17:574d

Afdeling Wiskunde, Faculteit der Exacte Wetenschappen. Vrije Universiteit Amsterdam, De Boelelaan 1081A, 1081 HV Amsterdam, The Netherlands

E-mail address: ran@cs.vu.nl

$U R L:$ http://www.cs.vu.nl/ ran

Afdeling Wiskunde, Faculteit der Exacte Wetenschappen. Vrije Universiteit Amsterdam, De Boelelaan 1081a, 1081 HV Amsterdam, The Netherlands

E-mail address: mcreurin@cs.vu.nl

$U R L:$ http://www.cs.vu.nl/ mcreurin 